SHORT COMMUNICATION

\title{
Detection of Poorly-Oriented Component in Uniaxially Stretched Poly(glycolic acid) Fiber Studied Using ${ }^{13}$ C Solid-State NMR
}

\author{
By Sokei SeKINE,,${ }^{1,2 * *}$ Wataru SAKIYAMA,${ }^{1}$ Kazuo YAMAUCHI,${ }^{1}$ and Tetsuo ASAKURA ${ }^{1}$
}

\section{KEY WORDS: Poly(glycolic acid) $/{ }^{13} \mathrm{C} \mathrm{CP} / \mathrm{MAS}$ NMR / ${ }^{13} \mathrm{C} \mathrm{CP}$ NMR / Oriented Structure /}

Poly(glycolic acid) (PGA) is the simplest and semi-crystalline aliphatic polyester. The uniaxially stretched PGA fiber has been successfully used as bio-absorbable suture materials for closing surgical wounds because of the high biodegradability and biocompatibility. ${ }^{1-3}$

However, one of the main disadvantages of PGA fiber is narrow window of processing conditions and therefore this suture has been used as a multi filament made of yarn which bound some of thin fibers. In order to improve such a problem, it seems important to accumulate the structural information on uniaxially stretched PGA fiber in atomic level. X-Ray diffraction study reported planar zig-zag structure for the well-oriented PGA sample ${ }^{4}$ and ${ }^{1} \mathrm{H}$ NMR relaxation studies showed the presence of the heterogeneous structure. ${ }^{5,6}$

In this communication, ${ }^{13} \mathrm{C} \mathrm{CP} \mathrm{NMR} \mathrm{spectra} \mathrm{of} \mathrm{un-stretched} \mathrm{and}$ uniaxially stretched PGA fibers were reported, where the spectra were obtained as a function of the angle between the fiber axis and external magnetic field. This gives a detailed information on the heterogeneous structures of the oriented PGA fibers by analyzing angle-dependent spectra on the basis of the theory on the chemical shift tensor transformations. ${ }^{7,8}$ The change in the physical properties of these PGA fibers could be interpreted with change in the heterogeneous structures, especially the fraction of poorly-oriented components.

\section{EXPERIMENTAL}

PGA was synthesized by Mitsui Chemicals, Inc. and its molecular weight was determined about $2 \times 10^{5}$ using GPC method. The PGA fiber was prepared at $230^{\circ} \mathrm{C}$ from the molten state. After cooling at room temperature, the fibers were stretched by $1.5,2.0,2.5$ and 3.0 times with a stretching rate of $100 \mathrm{~mm} / \mathrm{min}$, resulting fibers were heated again at a temperature just above the glass transition temperature of PGA. The blocks of the macroscopically aligned fibers were prepared carefully. The angledependent CP NMR spectra were observed using home made probe head prepared by us. A Bruker AVANCE-400 spectrometer was used at $100.6 \mathrm{MHz}$ for ${ }^{13} \mathrm{C}$ nuclei at $30^{\circ} \mathrm{C}$.

\section{RESULTS AND DISCUSSION}

The ${ }^{13} \mathrm{C}$ CP NMR spectra of the blocks of uniaxially stretched PGA fibers with different stretching ratios were summarized in Figure 1 when the angle of the fiber axis and the external magnetic field was $0^{\circ}$, that is, the fiber axis was parallel to the magnetic field. In order to examine change in the line shape at the lower field region of the spectra among the stretched samples carefully, the size of the line shape at the higher field region in each spectrum was adjusted in Figure 1.

With increasing stretching ratio, the shape of the lower field peak tends to be sharp. The remarkable change was observed between 2.0 and 2.5 times. Namely, a well-oriented component was observed when the stretching ratio is larger than 2.5 times. In order to obtain further

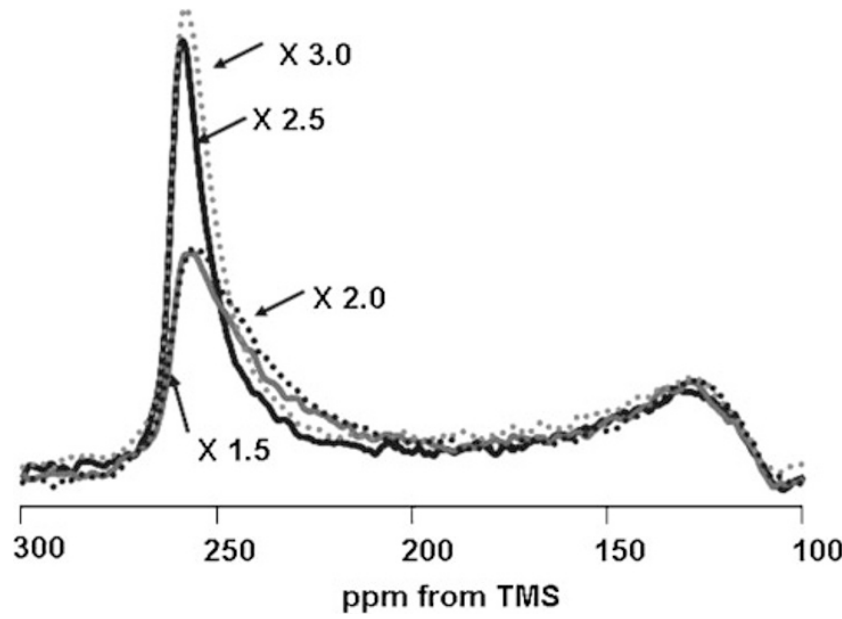

Figure 1. ${ }^{13} \mathrm{C} C P$ NMR spectra ( $\mathrm{C}=\mathrm{O}$ region) of the blocks of uniaxially stretched PGA fibers with different stretching ratio, $1.5(-)$, 2.0(---), 2.5(-) and 3.0(--). The angle of the fiber axis and the magnetic field was set to be $0^{\circ}$. The line shape of the higher field region was superimposed to emphasize change in the line shape of the lower field region among these samples.

information, a series of ${ }^{13} \mathrm{C}$ CP NMR spectra of PGA fiber blocks were observed as a function of the angle between the fiber axis and magnetic field. As an example, a series of the spectra of the stretched fiber block with stretching ratio, 3.0 were shown in Figure 2. The ${ }^{13} \mathrm{C} \mathrm{CP}$ NMR spectra change largely depending on the angle as is expected. Simulation of the spectra on the basis of orientation-dependent spin interaction tensors ${ }^{7}$ indicates the presence of three components in the oriented fibers, that is, two components (well-oriented (1) and poorly-oriented (2)) ones in the oriented region and another component (angle-independent powder pattern). From the simulation, the structural parameters, $\alpha_{\mathrm{F}}, \beta_{\mathrm{F}}$ and $p$ were also determined for each oriented component. Here, $\alpha_{\mathrm{F}}$, and $\beta_{\mathrm{F}}$ are defined as follows. To transform the ${ }^{13} \mathrm{C}$ chemical shift anisotropy (CSA) tensor from the principal axis system (PAS: $\sigma_{11}, \sigma_{22}, \sigma_{33}$ ) representation to the fiber axis system (FAS: $x, y, z$ ) representation, the ${ }^{13} \mathrm{C}$ CSA tensor is first rotated about $\sigma_{33}$ by $\alpha_{\mathrm{F}}$ to bring $\sigma_{22}$ into the $x y$ plane, followed by a rotation about $\sigma_{22}$ by $\beta_{\mathrm{F}}$ to bring $\sigma_{33}$ coincident with $z$. The width parameter, $p$ is defined as standard deviation of distribution of the fiber axis. Figure 2 shows that all the angle-dependent spectra of the stretched fibers with stretching ratio, 3.0 were well-reproduced by the simulation. The parameters determined here were summarized in Table I.

With increasing stretching ratio, the fraction of the angle-independent powder pattern component decreases, but no significant change between 2.0 and 2.5 times. However, as shown in Figure 1, a large spectral change

\footnotetext{
${ }^{1}$ Department of Biotechnology, Tokyo University of Agriculture and Technology, Koganei 184-8588, Japan

${ }^{2}$ Mitsui Chemical Analysis \& Consulting Service, Inc., 580-32, Nagaura, Sodegaura 299-0265, Japan

*To whom correspondence should be addressed (Tel: +81-438-64-2406, Fax: +81-438-64-2407, E-mail: Sokei.Sekine @ mitsui-chem.co.jp).
} 


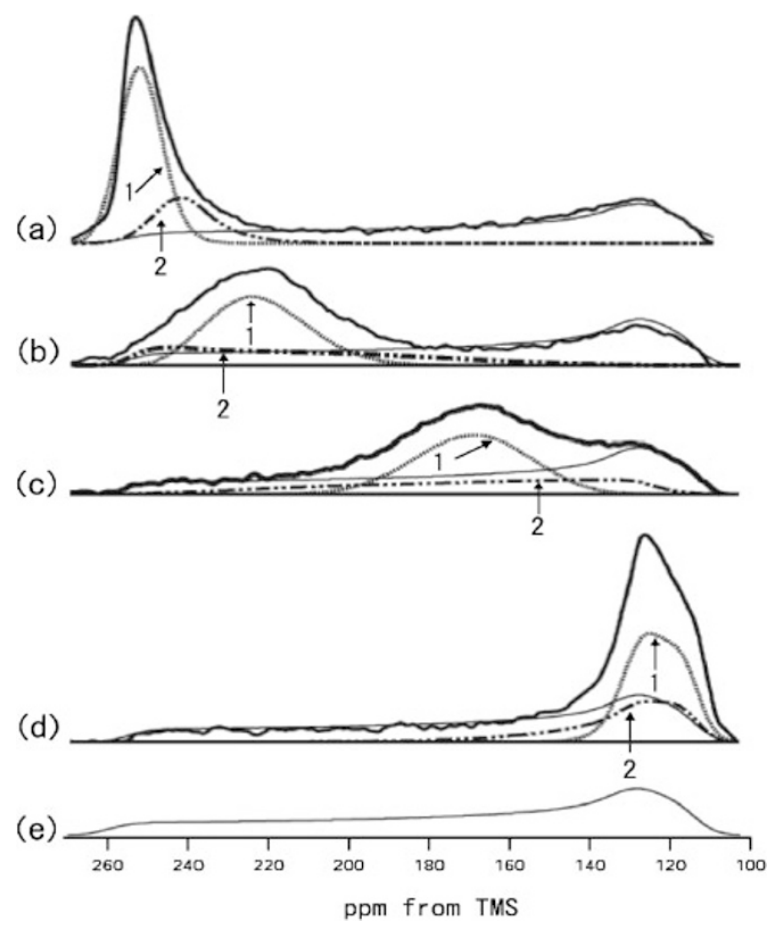

Figure 2. A series of ${ }^{13} \mathrm{C} C P$ NMR spectra ( $C=O$ region) of uniaxially stretched $P G A$ fibers with stretching ratio, 3.0. The angles between the axis of macroscopically aligned fibers and external magnetic field were changed as $0^{\circ}(\mathrm{a}), 30^{\circ}(\mathrm{b}), 55^{\circ}(\mathrm{c})$ and $90^{\circ}(\mathrm{d})$. The simulations were performed by assuming three components, 1: well-oriented (--- -), 2: poorly-oriented (.-.-.) and powder pattern component $(-) .{ }^{7}(\mathrm{e})$ is the ${ }^{13} \mathrm{C} \mathrm{CP}$ NMR spectrum of un-stretched PGA fiber.

was observed between 2.0 and 2.5 times. Thus, the change is due to structural change in the oriented region in the sample. With increasing stretching ratio, the fraction of well-oriented components increased linearly, but change in the fraction is not linear for the poorly-oriented component, 2. Especially, a remarkable decrease from 0.27 to 0.20 was obtained between 2.0 and 2.5 times for the poorly-oriented component. There are a similar tendency for the parameter, $p$; a remarkable decrease from 20 to 11 was also obtained between 2.0 and 2.5 times although only small change was obtained between 1.5 and 2.0 times, and also between and 2.5 and 3.0 times. Thus, the remarkable spectral change between 2.0 and 2.5 times in Figure 1 is concluded due to a remarkable change in the fraction of poorly-oriented region.

As mentioned above, the Euler angle, $\beta_{\mathrm{F}}$ is defined as the angle between the fiber axis and the direction of the chemical shift tensor, $\sigma_{33} .{ }^{7}$ The latter direction is approximately perpendicular to the $\mathrm{C}=\mathrm{O}$ bond direction within the plane of the $(\mathrm{C}=\mathrm{O})-\mathrm{O}$ group. From the $\mathrm{X}$-ray diffraction data reported by Chatani et al. ${ }^{4} \beta_{\mathrm{F}}$ was calculated to be about $5^{\circ}$ for the PGA chains in the crystalline sample. The PGA chains studied by their X-ray analysis correspond to the well-oriented PGA chains with stretching ratio, 3.0 in this study (Table I), where $\beta_{\mathrm{F}}$ was $0^{\circ}-5^{\circ}$ with small $p$ value, $6-8$. On the other hand, in the poorly-oriented region, $\beta_{\mathrm{F}}$ was $15-20^{\circ}$ with relatively wide distribution of the fiber axis, $p=11-20$.

\begin{tabular}{|c|c|c|c|c|c|}
\hline \multirow{2}{*}{ Component } & & \multicolumn{4}{|c|}{ Stretching ratio } \\
\hline & & $\times 1.5$ & $\times 2.0$ & $\times 2.5$ & $\times 3.0$ \\
\hline \multirow[t]{4}{*}{ 1: well-oriented } & $\alpha_{F}$ & 90 & 90 & 90 & 90 \\
\hline & $\beta_{\mathrm{F}}$ & 5 & 5 & 0 & 0 \\
\hline & $p$ & 7 & 8 & 6 & 6 \\
\hline & fraction & 0.15 & 0.20 & 0.28 & 0.35 \\
\hline \multirow[t]{4}{*}{ 2: poorly-oriented } & $\alpha_{\mathrm{F}}$ & 90 & 90 & 90 & 90 \\
\hline & $\beta_{\mathrm{F}}$ & 18 & 20 & 15 & 17 \\
\hline & $p$ & 18 & 20 & 11 & 12 \\
\hline & fraction & 0.28 & 0.27 & 0.20 & 0.20 \\
\hline \multicolumn{2}{|c|}{ 3: powder pattern fraction } & 0.57 & 0.53 & 0.52 & 0.45 \\
\hline
\end{tabular}

It is interesting to observe thermal properties of these uniaxially stretched PGA fibers with different stretched ratio in relation with the structural analysis in this study. The glass transition temperature was $48.6^{\circ} \mathrm{C}, 49.3{ }^{\circ} \mathrm{C}, 50.7^{\circ} \mathrm{C}$ and $50.5^{\circ} \mathrm{C}$ for the stretched PGA fiber with the stretching ratio, 1.5, 2.0, 2.5 and 3.0, respectively. Thus, these temperatures change remarkably between 2.0 and 2.5 times. In general, PGA fiber with more than 2.5 times of the stretching ratio has been used as a suture material because the fiber after hydrolytic acceleration test was not strong at less than 2.5 times. In conclusion, the control of such a poorlyoriented component in the process of suture production seems very important.

Acknowledgment. T. A. acknowledges support from Promotion of Basic Research Activities for Innovative Biosciences, Japan. The authors are also grateful to Dr. H. Akieda for offering PGA fiber. K.Y. acknowledges the financial support of SENTAN JST for the probe head development.

Received: March 3, 2009

Accepted: April 15, 2009

Published: June 3, 2009

\section{REFERENCES}

1. C. Nakafuku and H. Yoshimura, Polymer, 45, 3583 (2004).

2. J. Blomqvist, B. Mannfors, and L. O. Pietila, Polymer, 43, 4571 (2002).

3. D. K. Gilding and A. M. Reed, Polymer, 20, 1459 (1979).

4. Y. Chatani, K. Suehiro, Y. Okita, H. Tadokoro, and K. Chujo, Makromol. Chem., 113, 215 (1968).

5. H. Montes de Ocaa, I. M. Warda, P. G. Kleina, M. E. Riesa, J. Roseb, and D. Farrarb, Polymer, 45, 7261 (2004).

6. S. Sekine, H. Akieda, I. Ando, and T. Asakura, Polym. J., 40, 10 (2008).

7. L. K. Nicholson, T. Asakura, M. Demura, and T. A. Cross, Biopolymers, 33, 847 (1993).

8. T. Asakura, T. Konakazawa, M. Demura, T. Ito, and Y. Maruhashi, Polymer, 37, 1965 (1996). 